\title{
Noise Effect on Thin Film Characterization Using Rotating Polarizer Analyzer Ellipsometer
}

\author{
T.M. El-AGEZ AND S.A. TAYA* \\ Physics Department, Islamic University of Gaza, P.O. Box 108, Gaza, Palestinian Authority \\ (Received December 21, 2010; in final form February 22, 2012)

\begin{abstract}
We present theoretically the characterization of $100 \mathrm{~nm} \mathrm{SiO} 2$ thin film using spectroscopic rotating polarizer analyzer ellipsometer in which the two elements are rotating synchronously in opposite directions with the same angular speed. The proposed sample consists of air (ambient) $/ \mathrm{SiO}_{2}$ (thin film)/Si (substrate). The ellipsometric parameters $\psi$ and $\Delta$ are calculated when a clean signal is received by the detector and when a hypothetical noise is imposed on this signal. The film thickness and the optical constants of the film are calculated for the noisy signal in the spectrum range $200-800 \mathrm{~nm}$. The results are compared with the proposed thickness and with the published values for $\mathrm{SiO}_{2}$ optical constants.
\end{abstract}

PACS: 07.60.Fs, 78.66.Bz, 78.20.Ci

\section{Introduction}

Ellipsometry is a very sensitive optical technique for the study of optical properties of thin films. It is based on the measurement of the change in the light polarization state after reflection from a sample. Ellipsometry has received an increasing interest [1-5] because of several reasons: it is nondestructive technique, has an outstanding sensitivity to minute interfacial changes, can be used for in situ measurements, and can be operated at different wavelengths and under multiple angles of incidence.

Two parameters $\psi$ (the ratio of reflection coefficients) and $\Delta$ (phase change between $p$ - and $s$-polarized lights) are determined in ellipsometric measurements. The parameters $\psi$ and $\Delta$ are given by

$$
\rho=\frac{r_{p}}{r_{s}}=\tan (\psi) \mathrm{e}^{\mathrm{i} \Delta},
$$

where $r_{p}$ and $r_{s}$ are the complex Fresnel reflection coefficients for the $p$ and $s$ components which may be written as

$$
\left\{\begin{array}{l}
r_{p}=\rho_{p} \mathrm{e}^{\mathrm{i} \delta_{p}}, \\
r_{s}=\rho_{s} \mathrm{e}^{\mathrm{i} \delta_{s}},
\end{array}\right.
$$

where $\delta_{p}$ and $\delta_{s}$ are the phase changes for the $p$ and $s$ components of light.

The scanning ellipsometer has shown a great accuracy in determining optical constants of materials in the range $1.5-6 \mathrm{eV}$ photon energy $[6,7]$. Consequently ellipsometry has been a hot topic of research and the technique has been proposed and constructed in different configurations [8-12]. Among the various configurations of spectroscopic ellipsometers commonly used is the rotating analyzer ellipsometer (RAE) [6]. The RAE has the advantage of simple system design but it involves the dc component which causes a serious problem. The reduction of the dc background requires particular techniques

\footnotetext{
* corresponding author; e-mail: staya@iugaza.edu.ps
}

and the calibration of such system is also time consuming [13]. Azzam [14] suggested synchronously rotating both the polarizer and the analyzer with the ratio 1:3. In his work, the intensity of light reaching the detector includes four cosine and four sine terms. His system suffers a lack of clear explanation of each coefficient and its relation to the ellipsometric parameters $\psi$ and $\Delta[10]$. A RPAE in which the speed ratio between the rotating elements is 1:2 was proposed and constructed by Chen and Lynch [10]. In this design, the errors arising from the phase shift and dc background are eliminated. The same group suggested improving this RPAE [11, 12] with the incident angle being fully variable. Moreover, a fixed polarizer was placed in the optical path to eliminate the source polarization effect.

Recently, a spectroscopic RPAE has been proposed in which the rotating elements have the same angular speed but in opposite directions [15]. The intensity emerging from the rotating analyzer contains four components, one $\mathrm{dc}$ and three cosine terms, with frequencies of $\omega, 2 \omega$, and $3 \omega$. The main advantage of the proposed ellipsometer is that: it is feasible to extract the ellipsometric parameters $\psi$ and $\Delta$ from the even Fourier coefficients without relying on the dc component which is considered to be a serious problem in a rotating-analyzer or -polarizer ellipsometer. This allows measurements in semidark room without worrying about stray light problems, dark currents in detectors, and long term fluctuations in light sources. The calculations of the optical parameters of c-Si, $\mathrm{Au}$, and GaAs were in agreement with the published data.

In this work, we examine theoretically the noise effect on the characterization of $100 \mathrm{~nm} \mathrm{SiO}_{2}$ thin film using the RPAE described in [15]. The structure under consideration consists of a thin $\mathrm{SiO}_{2}$ film sandwiched between air and silicon substrate. The optical parameters and the thickness of the film are calculated from the ellipsometric parameters and compared to accepted values. In order to simulate reality we consider that a hypothetical noise is 
imposed on the clean signal. This noise causes a percent error on the calculated values. The percent error is also presented.

\section{Theory}

The RPAE assumed in the present work comprises light source, fixed linear polarizer with an azimuth angle $\theta$, linear polarizer rotating at an angular speed $\omega$, a sample, linear analyzer rotating at an angular speed $\omega$ but in opposite direction to that of the polarizer, and photodetector. A schematic diagram of the proposed ellipsometer is shown in Fig. 1. The azimuth angles of the rotating polarizer and the rotating analyzer are given by $P=\omega t+\tau$ and $A=-\omega t+\delta$, respectively, where $\tau$ and $\delta$ are the azimuth angles of the rotating polarizer and the rotating analyzer at $t=0$, respectively. The azimuth angles $\theta, P$, and $A$ are referred to $p$-axis of polarization.

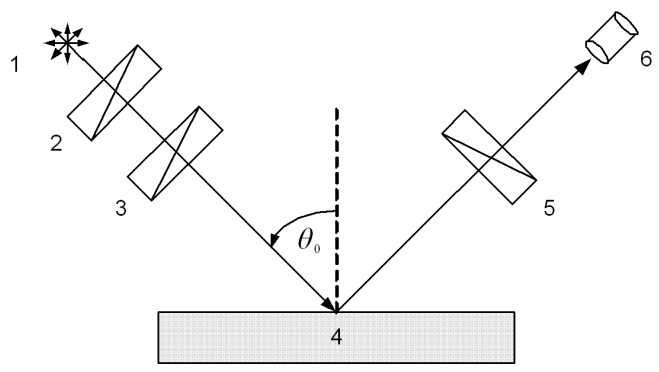

Fig. 1. Simplified schematic diagram of the RPA ellipsometer, (1) unpolarized light, (2) fixed linear polarizer,

(3) linear polarizer rotating at $\omega,(4)$ isotropic sample,

(5) linear analyzer rotating at $\omega$, and (6) detector.

We here assume $\mathrm{SiO}_{2}$ film of thickness $d=100 \mathrm{~nm}$ and refractive index $N_{1}$ sandwiched between an ambient (air) of refractive index $N_{0}$ and a c-Si substrate of refractive index $N_{2}$ as shown in Fig. 2. The Fresnel reflection coefficients of the proposed structure are given by [1]:

$$
r_{j}=\frac{r_{01 j}+r_{12 j} \exp (-\mathrm{i} 2 \beta)}{1+r_{01 j} r_{12 j} \exp (-\mathrm{i} 2 \beta)},
$$

where $j$ stands for $s$ in $s$-polarization and for $p$ in $p$-polarization,

$$
\begin{aligned}
& r_{01 s}=\frac{N_{0} \cos \left(\theta_{0}\right)-N_{1} \cos \left(\theta_{1}\right)}{N_{0} \cos \left(\theta_{0}\right)+N_{1} \cos \left(\theta_{1}\right)}, \\
& r_{12 s}=\frac{N_{1} \cos \left(\theta_{1}\right)-N_{2} \cos \left(\theta_{2}\right)}{N_{1} \cos \left(\theta_{1}\right)+N_{2} \cos \left(\theta_{2}\right)}, \\
& r_{01 p}=\frac{N_{1} \cos \left(\theta_{0}\right)-N_{0} \cos \left(\theta_{1}\right)}{N_{1} \cos \left(\theta_{0}\right)+N_{0} \cos \left(\theta_{1}\right)}, \\
& r_{12 p}=\frac{N_{2} \cos \left(\theta_{1}\right)-N_{1} \cos \left(\theta_{2}\right)}{N_{2} \cos \left(\theta_{1}\right)+N_{1} \cos \left(\theta_{2}\right)}, \\
& \beta=\frac{2 \pi d N_{1} \cos \left(\theta_{1}\right)}{\lambda},
\end{aligned}
$$

and $\theta_{1}$ and $\theta_{2}$ are the refraction angles in the sample and the substrate, respectively.

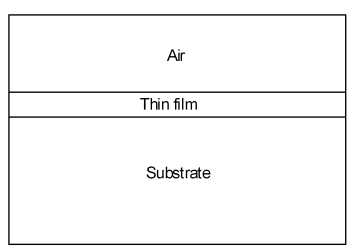

Fig. 2. Schematic diagram for the sample under consideration.

Ellipsometric theory can be formulated in terms of the Jones matrix formalism or the Mueller matrix formalism. The Jones matrix approach is usually adopted when a completely polarized light is assumed. When a significant amount of reflected or transmitted light becomes depolarised, it may be necessary to introduce the Mueller matrix representation. In this scheme, a $4 \times 4$ matrix connects the Stokes vectors representing the input and the output beams. In the present work, we adopt the Jones matrix formalism. In this case the transmitted electric field $E_{\mathrm{t}}$ in terms of the incident field $E_{\mathrm{i}}$ is then given by

$$
\begin{aligned}
E_{\mathrm{t}} & =\left[\begin{array}{cc}
\cos ^{2} A & \sin A \cos A \\
\sin A \cos A & \sin ^{2} A
\end{array}\right]\left[\begin{array}{cc}
r_{p} & 0 \\
0 & r_{s}
\end{array}\right] \\
& \times\left[\begin{array}{cc}
\cos ^{2} P & \sin P \cos P \\
\sin P \cos P & \sin ^{2} P
\end{array}\right]\left[\begin{array}{c}
\cos \theta \\
\sin \theta
\end{array}\right] E_{\mathrm{i}} .
\end{aligned}
$$

Performing the product of matrices in Eq. (9) and calculating the light intensity $I$ received by the detector as $E_{\mathrm{t}}^{\dagger} E_{\mathrm{t}}$ we obtain

$$
\begin{aligned}
I= & {\left[\frac{N}{2}+\frac{M\left(s_{1}+s_{2}\right)}{2}+\frac{N s_{1} s_{2}}{2}+\rho_{p} \rho_{s} s_{3} s_{4} \cos \Delta\right] } \\
& \times[1+\cos 2(\omega t+\tau-\theta)],
\end{aligned}
$$

where $N=\rho_{p}^{2}+\rho_{s}^{2}, M=\rho_{p}^{2}-\rho_{s}^{2}, s_{1}=\cos 2(\omega t+\tau), s_{2}=$ $\cos 2(-\omega t+\delta), s_{3}=\sin 2(\omega t+\tau), s_{4}=\sin 2(-\omega t+\delta)$, and $\Delta=\delta_{p}-\delta_{s}$. The amplitudes of the reflection coefficients in terms of $M$ and $N$ are given by

$$
\rho_{p}=\sqrt{\frac{N+M}{2}} \text { and } \rho_{s}=\sqrt{\frac{N-M}{2}} .
$$

The intensity at the detector in terms of the angular speed of the polarizer will be

$$
I(t)=a_{0}+\sum_{n=1}^{3} a_{n} \cos 2 n \omega t+\sum_{n=1}^{3} b_{n} \sin 2 n \omega t .
$$

Assuming that the azimuth angles $\theta, \tau$, and $\delta$ are equal to zero, Eq. (12) reduces to

$$
I(t)=a_{0}+a_{1} \cos \omega t+a_{2} \cos 2 \omega t+a_{3} \cos 3 \omega t,
$$
where

$$
\begin{aligned}
& a_{0}=\frac{5}{4} \rho_{p}^{2}+\frac{1}{4} \rho_{s}^{2}-\frac{1}{2} \rho_{p} \rho_{s} \cos \Delta \\
& a_{1}=\frac{15}{8} \rho_{p}^{2}-\frac{1}{8} \rho_{s}^{2}-\frac{1}{4} \rho_{p} \rho_{s} \cos \Delta \\
& a_{2}=\frac{3}{4} \rho_{p}^{2}-\frac{1}{4} \rho_{s}^{2}+\frac{1}{2} \rho_{p} \rho_{s} \cos \Delta \\
& a_{3}=\frac{1}{8} \rho_{p}^{2}+\frac{1}{8} \rho_{s}^{2}+\frac{1}{4} \rho_{p} \rho_{s} \cos \Delta
\end{aligned}
$$

The ellipsometric parameters $\psi$ and $\Delta$ can be obtained using any set containing three coefficients. In terms of $a_{1}$, $a_{2}$, and $a_{3}, \tan \psi$ and $\cos \Delta$ are given 


$$
\begin{aligned}
\tan \psi & =\frac{\sqrt{a_{1}+a_{3}}}{\sqrt{a_{1}-4 a_{2}+9 a_{3}}}, \\
\cos \Delta & =\frac{3 a_{3}-a_{1}+2 a_{2}}{\sqrt{\left(a_{1}+a_{3}\right)\left(a_{1}-4 a_{2}+9 a_{3}\right)}} .
\end{aligned}
$$

\section{Numerical calculations}

In the following calculations, we assume the incidence angle to be $70^{\circ}$ and the azimuth angles $\theta, \tau$, and $\delta$ are set to zero. Based on the published values for the refractive indices of c-Si and $\mathrm{SiO}_{2}$ [16], we calculate $r_{p}$ and $r_{s}$ using Eq. (3). Simulated light signals are generated based on Eq. (9). The Fourier transform of the generated signal was taken to extract the coefficients $a_{0}$ through $a_{3}$. Equations (18) and (19) are used to calculate the ellipsometric parameters $\psi$ and $\Delta$ in the spectrum range $200-800 \mathrm{~nm}$. In practical work, random fluctuations in the recorded signal appear due to the noise. This noise may originate from a variety of sources such as thermal fluctuations in the light source, mechanical vibrations, pickup of stray $\mathrm{AC}$ light, and the self-generated noise in detectors and electronics. In order to simulate real signals, noise was generated using MathCAD code and was superimposed on the clean signal according to the following equation:

$$
\begin{aligned}
& I_{\text {noise }}=[\operatorname{rnd}(c)-c / 2] I+[\operatorname{rnd}(e)-e / 2] \\
& \quad+0.0001 I_{\max },
\end{aligned}
$$

where MathCAD's $\operatorname{rnd}(c)$ function produces random noise in the range from 0 to $c$ and $\operatorname{rnd}(e)$ function produces random noise in the range from 0 to $e$. In our simulation, we consider $c=e=1$. The first term represents the random noise recorded by the detector due to thermal fluctuations of the light source after passing through the system, the second corresponds to the Johnson noise and shot noise encountered in the detector and readout electronics, and the third term represents the $\mathrm{dc}$ offset due to long time drifts. Figure 3 shows the noise superimposed on the clean signal.

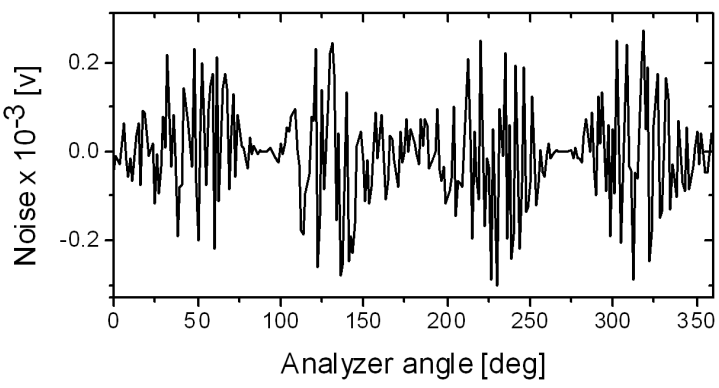

Fig. 3. The noise superimposed on the clean signal.

This noise is added to the pure signal. The Fourier transform of the noisy signal is taken to extract the new coefficients $a_{0}$ through $a_{3}$ in the presence of the noise. Equations (18) and (19) are used again to calculate the ellipsometric parameters $\psi$ and $\Delta$ for the noisy signal in the same spectrum range. Figures 4 and 5 show the calculated ellipsometric parameters $\psi$ and $\Delta$, respectively, for the clean and noisy signals.

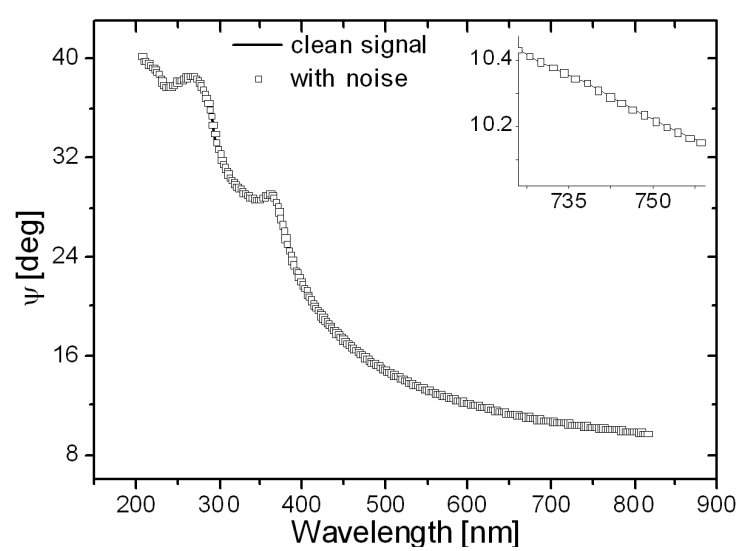

Fig. 4. The ellipsometric parameter $\psi$ as a function of the wavelength from 200 to $800 \mathrm{~nm}$.

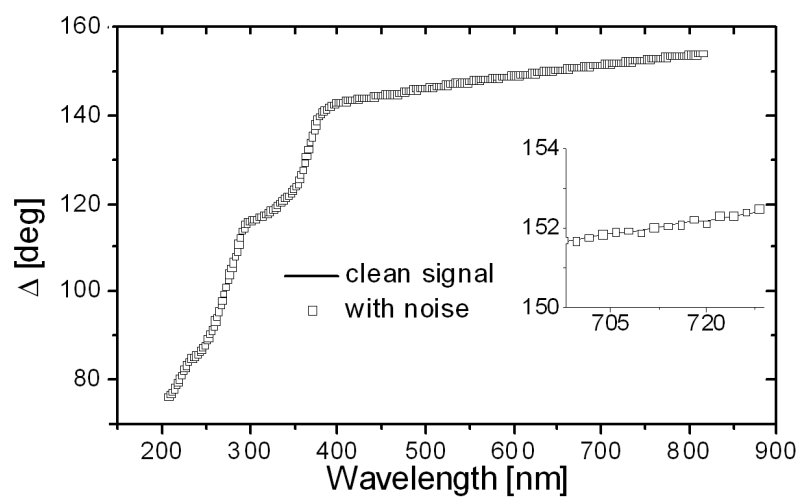

Fig. 5. The ellipsometric parameter $\Delta$ as a function of the wavelength from 200 to $800 \mathrm{~nm}$.

The percent error in the calculated values of $\psi$ and $\Delta$ is shown in Fig. 6. These fluctuations shown in the figure are due to the noise imposed on the clean signal as mentioned before. It is worth to mention that these values of $\psi$ and $\Delta$ were calculated using the coefficients $a_{1}, a_{2}$, and $a_{3}$ without depending on the dc offset of the signal for the clean and the noisy signals. If the dc term is considered in the calculations, the percent error in $\psi$ and $\Delta$ would be much higher [15]. This ellipsometer in which the polarizer and the analyzer rotate in the opposite directions with the same angular speed has the advantage that the results do not depend entirely on this term. As can be seen from Fig. 6 , the percent error in $\Delta$ is higher than that in $\psi$. The phase change $\Delta$ is critically dependent on the noise.

Making use of Eqs. (1) and (3), $\tan (\psi) \mathrm{e}^{\mathrm{i} \Delta}$ can be written as

$$
\begin{aligned}
\rho & =\tan (\psi) \mathrm{e}^{\mathrm{i} \Delta} \\
& =\frac{r_{01 p}+r_{12 p} \exp (-2 \mathrm{i} \beta)}{1+r_{01 p} r_{12 p} \exp (-2 \mathrm{i} \beta)} \frac{1+r_{01 s} r_{12 s} \exp (-2 \mathrm{i} \beta)}{r_{01 s}+r_{12 s} \exp (-2 \mathrm{i} \beta)} .
\end{aligned}
$$




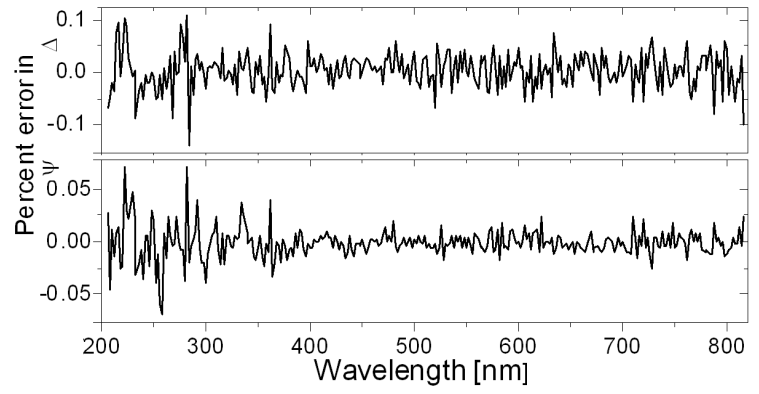

Fig. 6. Percent error in $\psi$ and $\Delta$ as a function of the wavelength. This error arises from the noise imposed on the signal.

Equation (21) can be simplified [17] to give a quadratic equation as

$$
A \xi^{2}+B \xi+C=0
$$

where

$$
\begin{aligned}
& A=\left(\rho r_{01 p}-r_{01 s}\right) r_{12 p} r_{12 s}, \\
& B=r_{01 p} r_{01 s}\left(\rho r_{12 p}-r_{12 s}\right)+\rho r_{12 s}-r_{12 p}, \\
& C=\rho r_{01 s}-r_{01 p},
\end{aligned}
$$

and

$$
\xi=\exp (-2 \mathrm{i} \beta) .
$$

The solutions of Eq. (22) have the conventional form

$$
\xi=\exp (-2 \mathrm{i} \beta)=\frac{-B \pm \sqrt{B^{2}-4 A C}}{2 A} .
$$

The thickness of the $\mathrm{SiO}_{2}$ film, $d$, in terms of $\xi, N_{1}$ and $\theta_{1}$ is

$$
d=\frac{i \lambda \ln (\xi)}{4 \pi N_{1} \cos \left(\theta_{1}\right)} .
$$

In the considered spectrum range, $\mathrm{SiO}_{2}$ is lossless. In this case the extinction coefficient is zero, i.e. $k=0$. Thus $\beta$ will be real and hence $\xi=\exp (-2 \mathrm{j} \beta)$ is pure complex. Therefore,

$$
\ln |\xi|=0 .
$$

Finally, the solution is to find the value of $N_{1}$ that makes

$$
\ln |\xi|=\ln \left|\frac{-B \pm \sqrt{B^{2}-4 A C}}{2 A}\right|=0 .
$$

A computer program was generated using MathCAD 2000 to solve Eq. (30) and to find the refractive index of the sample. Figure 7 shows the results obtained for the refractive index of $\mathrm{SiO}_{2}$ thin film using the ellipsometric parameters $\psi$ and $\Delta$ of the noisy signal as a function of the wavelength in the range $200-800 \mathrm{~nm}$. The figure also shows a comparison between accepted and calculated values of the film index. The percent error in the film refractive index as a function of the wavelength is shown in Fig. 8. This error arises from the noise imposed on the clean signal. The error ranges between $\pm 1.3 \%$ which is accepted range.

The film thickness can be calculated by substituting the calculated values of the film index into Eq. (29). The

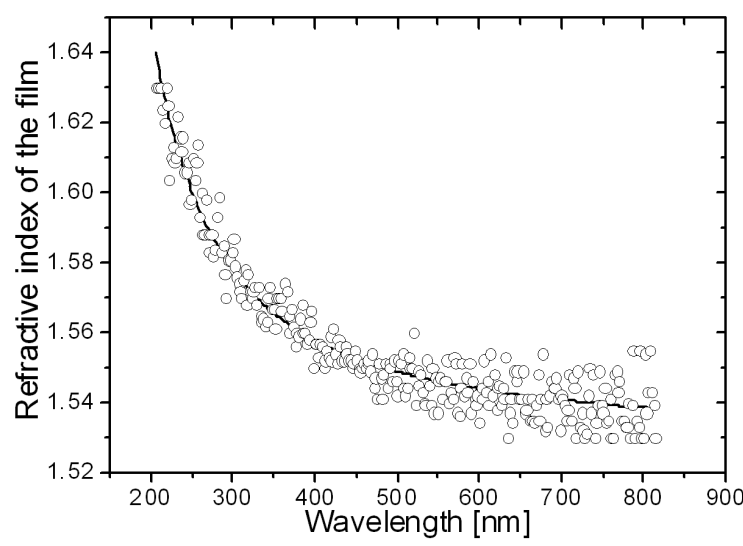

Fig. 7. Refractive index of the film versus the wavelength. Line: published values, points: calculated values with the noise.

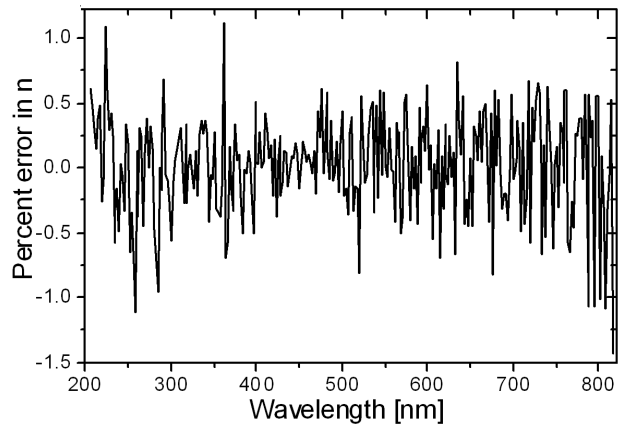

Fig. 8. Percent error in the film refractive index as a function of the wavelength.

results obtained for the thickness are plotted in Fig. 9 as a function of the wavelength. The percent error in the film thickness as a function of the wavelength is shown in Fig. 10. This error arises from the noise imposed on the clean signal. The error ranges between $\pm 2 \%$ which is accepted range.

It should be pointed out that in real situations it is not easy to align the optical elements with respect to the

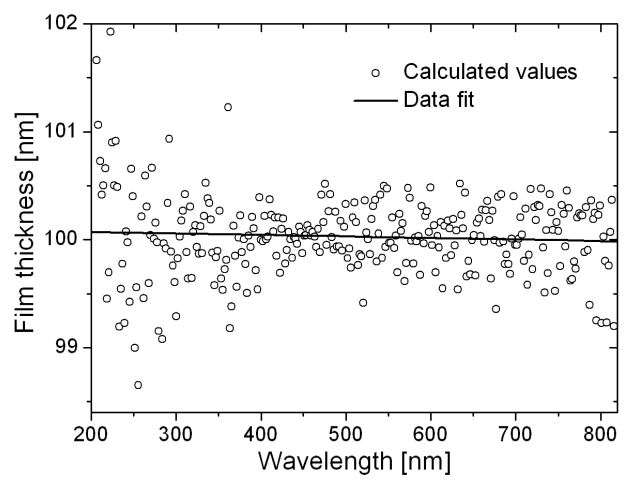

Fig. 9. Film thickness obtained from simulated data with noise as a function of the wavelength. 


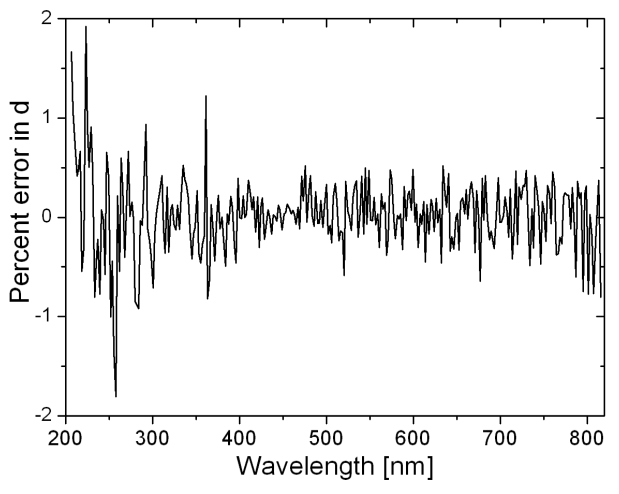

Fig. 10. Percent error in the film thickness as a function of the wavelength.
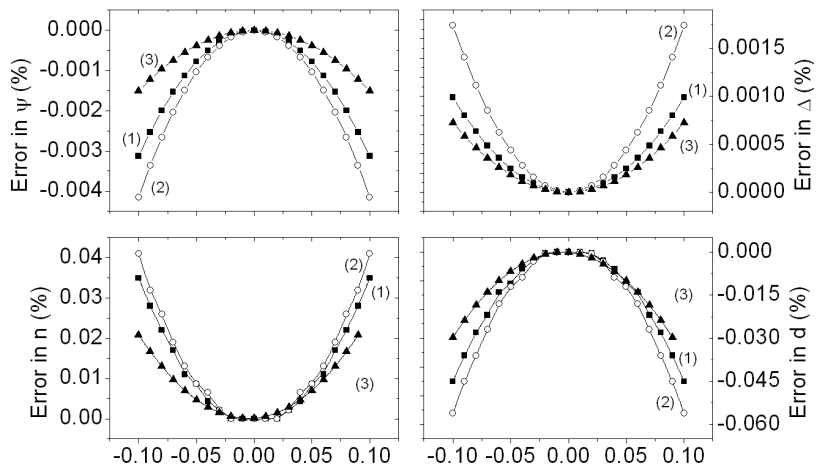

Fig. 11. Percent error in $\psi, \Delta, n$, and $d$, for $\mathrm{SiO}_{2}$ film at $\lambda=632.8 \mathrm{~nm}$ and $\theta_{0}=70^{\circ}$, as a function of the error in (1) $\theta,(2) \tau$, and (3) $\delta$ successively varied from $-0.1^{\circ}$ to $0.1^{\circ}$ in steps of $0.01^{\circ}$ while keeping the two other variables equal to zero.

plane of incidence. Azimuthal misalignment of optical elements is considered one of the main sources of systematic errors. Thus, it is important to study the effect of misalignment of these optical elements on the parameters extracted from the ellipsometric structure. The percent error in the ellipsometric parameters $\psi$ and $\Delta$ as well as the index of refraction $n$ and the film thickness $d$ are here studied as a function of the misalignment of the optical element. Figure 11 shows the percent error on $\psi, \Delta, n$, and $d$ as a function of the errors of the fixed polarizer azimuth angle $\theta$, the rotating polarizer azimuth angle $\tau$, and the rotating analyzer azimuth angle $\delta$ successively varied from $-0.1^{\circ}$ to $0.1^{\circ}$ in steps of $0.01^{\circ}$ while keeping the two other variables equal to zero. As can be seen from the figure, the impact of these errors on $\psi, \Delta, n$, and $d$ is not significant for small misalignment. The figure also reveals that the error due to misalignment of the azimuth angle $\delta$ has almost the lowest impact on the four parameters under consideration.

\section{Conclusion}

We have presented theoretically the characterization of $100 \mathrm{~nm} \mathrm{SiO}_{2}$ thin film using RPAE in which the polarizer and the analyzer rotate synchronously in opposite directions with the same angular speed. The film thickness and the optical constants of the film are calculated for the noisy signal in the spectrum range $200-800 \mathrm{~nm}$. As can be seen from the results, the proposed RPAE is very accurate and the percent error in the calculated optical parameters and thickness of the film is in the accepted range.

\section{References}

[1] R.M. Azzam, N.M. Bashara, Ellipsometry and Polarized Light, North-Holland, Amsterdam 1977.

[2] P.S. Hauge, Surf. Sci. 96, 108 (1980).

[3] J.A. Woollam, P.G. Snyder, M.C. Rost, Thin Solid Films 166, 317 (1988).

[4] K. Vedam, Thin Solid Films 313, 1 (1998).

[5] D.E. Aspnes, Thin Solid Films 455, 3 (2004).

[6] D.E. Aspnes, Opt. Commun. 8, 222 (1973).

[7] D.E. Aspnes, Appl. Opt. 4, 220 (1975).

[8] A.R. Zaghloul, R.M. Azzam, Surf. Sci. 96, 168 (1980).

[9] L. Vina, C. Umbatch, M. Cardona, L. Vodopyanov, Phys. Rev. B 29, 6752 (1984).

[10] L.Y. Chen, D.W. Lynch, Appl. Opt. 26, 5221 (1987).

[11] L.Y. Chen, X.W. Feng, Y. Su, H.Z. Ma, Y.H. Qian, Thin Solid Films 234, 385 (1993).

[12] L.Y. Chen, X.W. Feng, Y. Su, H.Z. Ma, Y.H. Qian, Appl. Opt. 33, 1299 (1994).

[13] C. Wijers, Appl. Phys. B 27, 5 (1982).

[14] R.M. Azzam, Opt. Commun. 25, 137 (1978).

[15] T.M. El-Agez, A.A. El Tayyan, S.A. Taya, Thin Solid Films 518, 5610 (2010).

[16] E.D. Palik, Handbook of Optical Constants of Solids, Academic Press, San Diego, CA 1985.

[17] S. Samuel, Surf. Sci. 56, 97 (1976). 